\title{
Calculations of three-dimensional magnetic normal modes in mesoscopic permalloy prisms with vortex structure
}

\author{
Ming Yan, Riccardo Hertel, and Claus M. Schneider \\ Institute of Solid State Research IFF-9, Jülich Research Center, D-52425 Jülich, Germany \\ (Received 10 July 2007; revised manuscript received 9 August 2007; published 13 September 2007)
}

\begin{abstract}
Static flux-closure structures in three-dimensional (3D) mesoscopic ferromagnets are known to differ quite significantly from their 2D counterparts. How these differences reflect in the dynamic properties of the magnetization is, to date, an open question. Micromagnetic simulations are employed to study the normal modes of magnetic oscillations in thick $(60-80 \mathrm{~nm})$ rectangular Permalloy prisms with 3D Landau-type flux-closure domain structure. Various magnetic normal modes are excited by a short field pulse and extracted using methods based on Fourier analysis. In particular, well-defined modes in the range of a few $\mathrm{GHz}$ are identified as oscillations of vortices, domain walls, and as excitations localized in the corners. The asymmetric Bloch wall in the center of the 3D Landau structure wall is a genuinely three-dimensional feature and thus gives rise to effects which were not reported in previous studies on 2D systems. It is argued that experimental evidence of these findings can be obtained.
\end{abstract}

DOI: 10.1103/PhysRevB.76.094407

PACS number(s): 75.75.+a, 75.40.Gb, 75.40.Mg, 76.50.+g

\section{INTRODUCTION}

Any memory device based on ferromagnetic elements operates with processes resulting from spin dynamics, which intrinsically determine the speed of the device. Typical dynamical processes occurring in ferromagnets after a perturbation of the static configuration are normal excitations or magnons. More complicated oscillations can often be either expanded in series of magnons in the linear case or studied in terms of interactions between magnons and other excitations in the nonlinear case. Due to the complexity of the ferromagnetic problem, however, the normal modes of bulk samples can be calculated analytically only in some limiting situations. For submicron confined magnetic elements, in which both the long-range dipolar and the short-range exchange interaction have to be taken into account, analytical solutions are only available for a few simple geometries. Alternatively, micromagnetic simulations combined with Fourier analysis methods have been proven to be an effective way to extract normal modes in confined magnetic structures. ${ }^{1,2}$ Usually, the normal modes obtained from simulations can be directly compared to the results of Brillouin light scattering (BLS), time-resolved (TR) magneto-optical Kerr studies (MOKE), and other experiments, which are currently the most suitable techniques to the study of magnetic normal modes experimentally. Excellent agreements are achieved in most of the cases.

Several studies on magnetic excitations in various thinfilm elements have been reported, including wires, ${ }^{3-7}$ rectangles, ${ }^{4,8-10}$ circular disks ${ }^{2,11-19}$ and squares ${ }^{20-24}$ with closure domains, rings, ${ }^{25}$ and several other elementary shapes. In all examples listed above, the samples studied were essentially two-dimensional (2D), i.e., thickness effects are not important as far as the profile of the dynamic mode is concerned. Along the sample thickness, the magnetization is assumed to be homogeneous, while it varies at the surface. Thus, the mode profiles in those systems can be considered to be confined on the sample surface. Recently, studies of magnetic excitations in 3D cylindrical dots ${ }^{26,27}$ and dc spin- polarized current-driven modes in $3 \mathrm{D}$ elliptical $\operatorname{dots}^{28}$ have been reported, pointing out the importance of threedimensional processes in spin dynamics.

In this paper, we present micromagnetic simulations of normal modes in 3D soft-magnetic rectangular samples. The thickness of the sample is in the range of $60-80 \mathrm{~nm}$ and the lateral extension of the order of a few $100 \mathrm{~nm}$. The thickness effect is already significant in the equilibrium configuration, leading to a characteristic, asymmetric magnetic ground state. This 3D character of both the sample and the magnetic ground state represents a fundamental qualitative difference compared with the numerous studied previously reported on $2 \mathrm{D}$ systems. In contrast to the $2 \mathrm{D}$ case, where the magnetic structure of a patterned element usually maintains the symmetry of the sample itself, with the same symmetry being evident in the corresponding normal modes, in the case of 3D elongated elements the magnetic ground state generally does not maintain the symmetry of the sample shape. The ground state is often a complicated magnetic-flux-closure domain pattern with an asymmetric Bloch wall separating the two main domains. ${ }^{29,30}$ As will be shown later, the asymmetric domain wall can be considered as a part of a stretched magnetic vortex core that extends through the thickness of the sample. Recently, the dynamics of magnetic vortices and vortex cores in thin-film elements has been intensively studied. ${ }^{2,11-24}$ In this context, the present study of the dynamics of a 3D vortex structure can be considered as an extension of the previous knowledge on this topic.

In our simulations we find that the excitation of a very large number of normal modes is typical for 3D samples. It is due to this mode complexity that usually only a few of them can be well resolved and understood. All the well-defined modes are three-dimensional, asymmetric excitations. We find in several respects a distinctly different behavior of these 3D modes as compared with that known from 2D elements. The purpose of this paper is to identify the most important 3D modes and to demonstrate the direct influence of the three-dimensionality of the sample and the resulting asymmetry of the magnetic ground state on the dynamic normal modes. 


\section{MICROMAGNETIC MODEL AND SIMULATION METHOD}

This study was carried out using 3D micromagnetic simulations. The simulation procedure is divided into two parts, the static and the dynamic one. In the static part, the ground state is obtained by energy minimization. The principle behind this is that equilibrium magnetic structures are minimum-energy configurations. Numerically, the discretized vector field of the magnetization can thus be varied systematically to minimize the total energy and thereby obtain a discretized representation of a stable magnetic configuration. In micromagnetism, the total energy of the system usually contains four terms: The Zeeman energy, the exchange energy, the dipolar energy, and the anisotropy energy. In our case, we study Permalloy samples-which have very small anisotropy - at zero static field. Therefore, only the exchange energy and dipolar energy are relevant to our static calculations. By representing the magnetization inside the sample as a vector field $\vec{M}(\vec{r})$ with the constraint $|\vec{M}|=M_{s}$ =const, where $M_{S}$ is the saturation magnetization, the two energy terms can be written as ${ }^{29}$

$$
E_{\mathrm{exc}}=\int_{(V)} A\left[\left(\nabla m_{x}\right)^{2}+\left(\nabla m_{y}\right)^{2}+\left(\nabla m_{z}\right)^{2}\right] d V
$$

and

$$
E_{\text {dip }}=-\frac{\mu_{0}}{2} \int_{(V)} \vec{M} \cdot \vec{H}_{s} d V,
$$

respectively. In Eq. (1), $A$ is the exchange constant and $m_{x}$, $m_{y}$, and $m_{z}$ are the three Cartesian components of the unit vector $\vec{m}=\vec{M} / M_{s}$. In Eq. (2), $\vec{H}_{s}$ is the dipolar field, which can be calculated by solving the Poisson equation $\Delta U=\nabla \vec{M}$ for the magnetic scalar potential $U$ using the well-known Neumann boundary conditions at the sample surface. The dipolar field is the gradient field of the potential $\left(\vec{H}_{S}\right.$ $=-\nabla U)$ and is a unique function of the magnetization distribution in the sample. The integrals of both Eqs. (1) and (2) are taken over the whole volume of the sample. The total energy of the system is uniquely determined by the magnetization distribution. ${ }^{29}$

The energy minimization method cannot be used to calculate dynamic oscillations of the magnetization as they occur, e.g., after a short perturbation by an external field pulse. To study these effects, the magnetization dynamics is modeled numerically by integrating the Landau-Lifshitz-Gilbert equation

$$
\begin{aligned}
\frac{d \vec{M}(\vec{r}, t)}{d t}= & -\frac{\gamma}{\left(1+\alpha^{2}\right)} \vec{M}(\vec{r}, t) \times \vec{H}_{\mathrm{eff}}(\vec{r}, t) \\
& -\frac{\alpha \gamma}{M_{s}\left(1+\alpha^{2}\right)} \vec{M}(\vec{r}, t) \times\left[\vec{M}(\vec{r}, t) \times \vec{H}_{\mathrm{eff}}(\vec{r}, t)\right],
\end{aligned}
$$

in time, where $\gamma$ is the gyromagnetic ratio, $\alpha$ is a phenomenological damping factor and $\vec{H}_{\text {eff }}$ is the effective field, which comprises external and internal field contributions from the various energy terms. The effective field is defined as the negative derivative of the total energy density with respect to the magnetization.

The static and the dynamic micromagnetic calculations are performed with our finite-element codes already used in previous studies. ${ }^{31,32}$ In this framework, the sample is discretized by dividing its volume into tetrahedral elements. Local values of each magnetization component, of the scalar magnetic potential $U$, and of each effective field contribution are defined at the nodal points of the elements. The magnetostatic field calculation is performed with a hybrid finiteelement-boundary-element method as described in Ref. 33. The static part of the calculation of the magnetic structure (i.e., the energy minimization) is performed using the conjugate gradient method. In this case, the nonlinear constraint $\left|M_{\mathrm{s}}\right|=$ const is observed automatically by representing the local magnetization direction with polar angles $\theta$ and $\phi$. In contrast to this, the dynamic integration uses the Cartesian components of the local magnetization. Minute variations of the magnitude of $|M|$, which may occur during the integration of the Gilbert equation, are constantly corrected by renormalizing the local magnetization at each node after a certain number of time steps. The numerical integration of Eq. (3) is performed using the Adams integration scheme. ${ }^{34}$

The simulations are performed for three rectangular Permalloy prisms with different thicknesses, i.e., 60, 70, and $80 \mathrm{~nm}$ to study the influence of the sample thickness on the normal modes. All prisms have the same aspect ratio $(2: 1: 0.4)$. In all cases, the cell size of the irregular tetrahedral mesh is approximately $5 \mathrm{~nm}$, which is fine enough to properly resolve all relevant inhomogeneous magnetic regions in the samples studied. The largest one $(400 \mathrm{~nm} \times 200 \mathrm{~nm}$ $\times 80 \mathrm{~nm})$ consists of 307200 tetrahedrons. Typical values for Permalloy $\left(M_{s}=1.0 \mathrm{~T}\right.$ and $\left.A=13 \mathrm{pJ} / \mathrm{m}, K=0\right)$ are used for the material parameters. The damping factor in the dynamic calculation is chosen to be $\alpha=0.01$, which is small enough to ensure underdamped precession of the magnetization as required to observe the characteristic normal modes.

\section{MODE EXCITATION AND FOURIER ANALYSIS}

The dynamic part of our study consists in first exciting magnetic oscillations by applying a short magnetic field pulse to the structure at equilibrium and extracting the various normal modes by means of Fourier analysis. The pulse is weak and short to ensure that the excitations stay in the linear regime. To couple to modes with different profiles, we have studied different situations, in which the field pulse is applied either in the plane of the sample (along the short axis of the prism) or perpendicular to it. In all cases we used a $10 \mathrm{mT}$ Gaussian-shaped field pulse with a width of $\sigma$ $=50 \mathrm{ps}$. After the sample magnetization is tipped away from equilibrium, the dynamic calculation yields the change of magnetization with time at every discretization point. To obtain a clear description of the oscillations, the static equilibrium structure is subtracted as a constant background from the results obtained from the dynamic calculation. The remaining vector field is then only the dynamic part of the magnetic structure, i.e., the variations of the magnetization in 


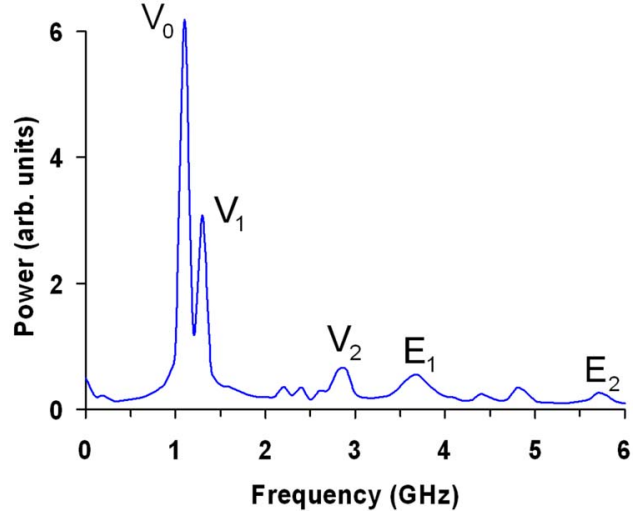

FIG. 1. (Color online) Power spectrum of $m_{z}$ for the 60-nm-thick sample excited by an in-plane field pulse. Each peak of the curve indicates a normal mode candidate.

time and space. The perturbation induced by the field pulse leads to rather noisy magnetization dynamics, from which the main oscillation frequencies can be extracted by means of Fourier transforms. Instead of using the spatially averaged magnetization, we calculate the Fourier transform at each node. Then, the total spectrum is obtained by averaging the amplitude of the oscillations over all nodes. In this way, the antisymmetric modes are obtained as well.

Figure 1 shows a typical power spectrum obtained by Fourier transform of the oscillations excited by a short field pulse. Experimentally, such spectra could be recorded, e.g., in ferromagnetic resonance (FMR) studies. Each peak of the spectrum represents a normal mode candidate, which can be analyzed individually. This is achieved by means of windowed Fourier transforms. First, the oscillations of the magnetization at each node are Fourier transformed. Then, a small frequency range of interest is selected and the oscillations with frequencies within this range are transformed back from the frequency domain to the time domain. By this, the spatiotemporal profile of each mode can be obtained separately, using both the amplitude and phase information. Similar methods were used in Refs. 7 and 9. The simulations are extended over a time period of at least $10 \mathrm{~ns}$ to obtain a frequency resolution (sampling) of $0.1 \mathrm{GHz}$ in the Fourier analysis. The marked peaks in Fig. 1 will be discussed later in detail as they correspond to magnetic normal modes which are well resolved in the calculation. They are connected with characteristic oscillations of the vortex, the domain walls, and the magnetization at the corners of the sample. These modes are all located in the magnetically inhomogeneous regions of the equilibrium structure.

For reasons of simplicity, we only focus on the variations of the $z$ component of the dynamic magnetization to analyze the spatial distribution of the normal modes, although the information on all three components is obtained. We elucidate the mode profiles by showing representative snapshots of the periodic oscillation, and we do this by displaying isosurfaces of the local magnitude of the oscillation. The dynamic evolution of these modes can be seen in various movies, which we provide as supplementary material. ${ }^{35}$ The analysis of the 3D modes is found to be significantly more

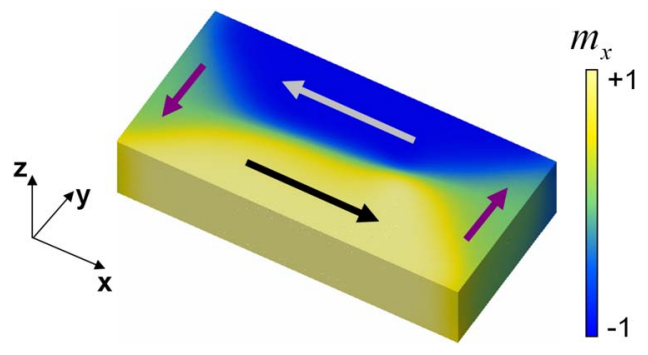

FIG. 2. (Color online) Magnetization distribution at equilibrium for the 60 -nm-thick sample. The arrows indicate the orientation of the magnetization in the four domains. The color (grayscale) represents the local value of $m_{x}$, i.e., the $x$ component of the magnetization.

complicated than in the $2 \mathrm{D}$ case. As will be shown, all the observed modes display markedly nontrivial features, even if they are clearly resolved in the simulations. The complicated spatial distribution of the modes is a direct consequence of the complexity of the 3D magnetic ground state. The visualization of the 3D mode profile also represents a difficulty; especially compared to the usual case of 2D modes, which can be easily displayed on a surface.

\section{THREE-DIMENSIONAL LANDAU STRUCTURE}

In all the cases that we have studied, the zero-field ground-state structure is an asymmetric, Landau-type 3D magnetic flux-closure domain pattern. Such an asymmetric structure was predicted by micromagnetic simulations of a rectangular Permalloy block ${ }^{30}$ and later observed experimentally in Fe particles. ${ }^{29}$ Since this ground-state structure is rather complex and important for the understanding of the corresponding normal modes, we give a brief description of it in this section.

Figure 2 shows the closure domain pattern on the surfaces of the 60-nm-thick sample. Here and in all the following cases, the coordinate system is chosen so that the $x$ axis is along the long axis of the slab, the $y$ axis along the intermediate edge, and $z$ along the thickness. The magnetization in the sample is divided into four domains, forming a Landau domain structure. In the 3D case, this basic flux-closure domain structure is not only inhomogeneous in the film plane, but also along the sample thickness. Hence, a study of the internal magnetic structure is essential for understanding its fundamental properties. Contrary to the 2D case, the 3D Landau structure is asymmetric. This asymmetry is already evident at the top surface shown in Fig. 2. Looking into the interior of the bar, it can be seen that the two large domains are separated by a characteristic domain wall known as asymmetric Bloch wall or Hubert-LaBonte wall. ${ }^{36,37}$ Figure 3 shows the magnetization distribution of the asymmetric Bloch wall on a cross section parallel to the $y-z$ plane in the middle of the sample. In the center of the sample, the magnetization changes its direction from one domain to another [Figs. 3(a) and 3(b)] by rotating in the $z$ direction [Fig. 3(d)], thus forming a Bloch type wall. Near the surfaces, however, the magnetization direction changes by rotating parallel to 

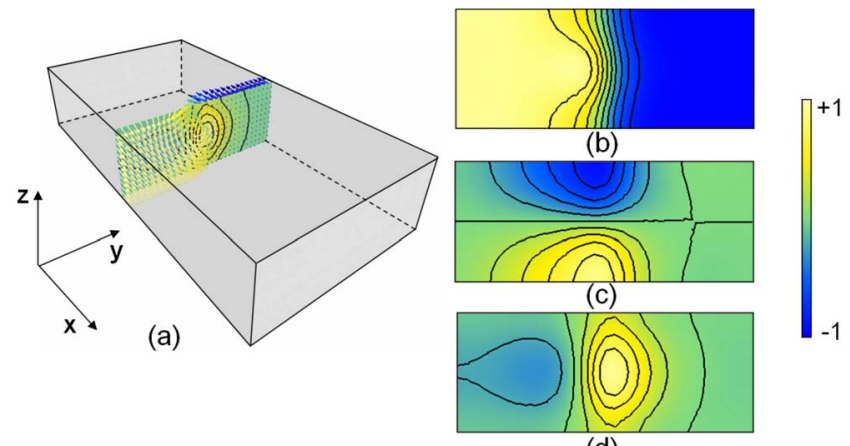

(d)

FIG. 3. (Color online) (a) Magnetization distribution indicated by arrows in a cross section parallel to the $y-z$ plane in the center of the 60-nm-thick sample. Color (grayscale) representations of $m_{x}$, $m_{y}$, and $m_{z}$ on this cross section are shown in (b), (c), and (d), respectively, to display the asymmetric Bloch wall and its Bloch and Néel components. Note that the domain wall is asymmetric and the Bloch component of the domain wall [yellow (bright) spot in panel (d)] is not exactly at the middle of the cross section.

the surface in the $y$ direction [see Fig. 3(c)], thereby forming the so-called Néel caps. This Néel-like transition at the surfaces minimizes the magnetostatic surface charges and thus the dipolar energy. It is clear that this structure cannot maintain the left-right symmetry of the sample.

Another important feature of the ground state is the formation of magnetic vortices on the top and bottom surfaces. The location of the vortex on the surface is determined by the orientation of the Néel cap, which can point in the positive or negative $y$ direction. On the surface, the caps constitute a $180^{\circ}$ Néel-type domain wall, which ends by connecting to the small closure domains with magnetization pointing along the $y$ direction, as shown in Fig. 2. If the magnetization in the Néel cap is parallel to that of the closure domain, these regions can be connected to each other smoothly. Otherwise, a vortex with a core perpendicular to the surface is formed at the intersection. ${ }^{38}$ Since the two Néel caps on the top and bottom surfaces have opposite orientation ${ }^{29}$ [see Fig. 3(c)] but the closure domain magnetization direction is the same on either surfaces, the vortices on the top and bottom surface are always located on opposite ends of the Néel wall.

The exact locations of the vortices on both surfaces of the 60-nm-thick sample are shown in Fig. 4(a). Thinking about two separate vortices can however be misleading. In fact, the structure could rather be considered as one stretched vortex, which is a distinct 3D effect. This can be seen from Fig. 4(b), which displays an isosurface with constant $m_{z}(0.7$ is chosen here). ${ }^{39}$ Inside the volume defined by this surface, $m_{z}$ has a larger value. The vortices on the surfaces are thus connected by a region with large $m_{z}$ inside the asymmetric Bloch wall. Obviously, different regions with perpendicular magnetization, namely, the central, Bloch-like part of the asymmetric domain wall and the cores of the vortices at the surface are directly connected. Therefore, a helpful and simpler alternative interpretation is to regard the entire tubelike region shown in Fig. 4(b) as a single, distorted vortex core. This interpretation is consistent with the feature of the asymmetric Bloch wall as discussed before. A minimum value of the

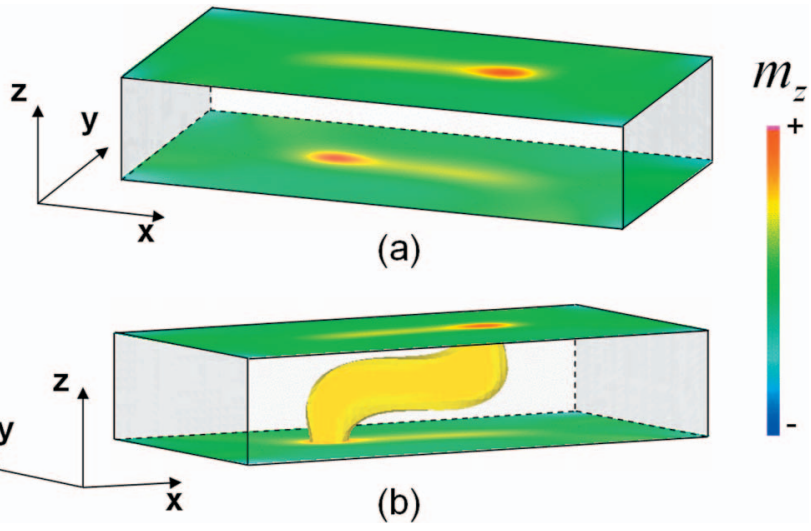

FIG. 4. (Color) (a) Vortex core positions on the top and bottom surface in equilibrium; indicated by the red spots (sample thickness: $60 \mathrm{~nm}$ ). (b) Isosurface displaying the area of constant $m_{z}$ value equal to 0.7 .

sample thickness is required to sustain such an asymmetric structure. In our calculations, we find that such a structure is formed in the 60-nm-thick sample, while a 50-nm-thick sample of the same aspect ratio yields vortices on the top and bottom that are almost overlapping. In this case, the ground state becomes similar to a symmetric closure domain pattern as is known in 2D systems. The aforementioned isosurface of $m_{z}$ then evolves towards a short tube oriented parallel to the $z$ axis, located in the center of the sample.

\section{THREE-DIMENSIONAL VORTEX CORE DYNAMICS}

The lowest-frequency mode is a relatively simple oscillation of the stretched vortex core region, which is closely related to the usual gyrotropic motion of the vortex core in a $2 \mathrm{D}$ system. This mode is labeled as mode $V_{0}$ in Fig. 1 . In thin-film elements, a displaced vortex core rotates with a characteristic frequency around its original location (usually the center of the system, but not in this case) due to a restoring force given by ${ }^{40}$

$$
\vec{F}=\vec{G} \times \frac{d \vec{R}}{d t}-\frac{\partial w(\vec{R})}{\partial \vec{R}},
$$

where $\vec{G}$ is the gyrovector with the same direction as the polarization of the vortex core $( \pm z), \vec{R}$ is the location of the vortex core, and $w$ is the potential energy of the system. From this equation, it can be seen that the displaced vortex should rotate counterclockwise if the vortex core is pointing in the positive $z$ direction. For disk-shaped thin-film elements with symmetric vortex structure, the gyrotropic frequency was shown to depend only on the aspect ratio of the sample. $^{41}$

Surprisingly, we found three variants of this gyrotropic mode occuring at different frequencies in our thick, 3D samples, unlike the previously reported singular gyrotropic mode in 2D cases, which occurs at only one well-defined frequency. The splitting of the gyrotropic mode into variants with different frequencies is therefore a genuinely three- 


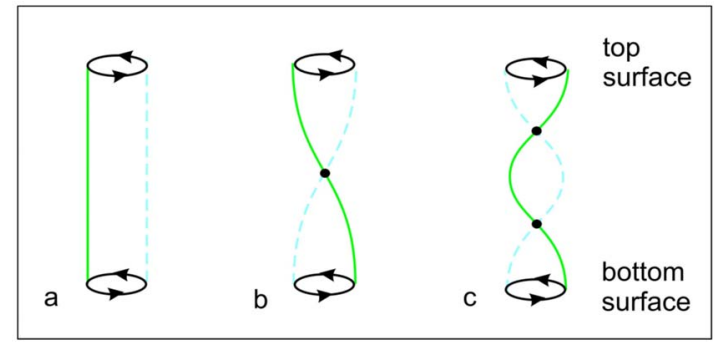

FIG. 5. (Color online) Schematic representation of three variants of the gyrotropic vortex mode in thick ferromagnets. The solid line indicates the vortex core position at a given time; the dashed line shows its position after half of the oscillation period. (a) Fundamental gyrotropic mode $\left(V_{0}\right)$, where the vortex core is rotating in the sample without variations along the sample thickness. (b) Firstorder mode $\left(V_{1}\right)$, characterized by a rotational core oscillation with a node in the center of the sample. (c) Second-order mode with two nodes $\left(V_{2}\right)$. In all cases, the vortices rotate in the same direction on both the top and the bottom surface.

dimensional effect. The occurrence of these new vortex modes can be easily understood by regarding the stretched vortex as an oscillating entity, which-contrary to the 2D case-contains interior degrees of freedom.

If we neglect for a moment the bending of the tubelike vortex core shown in Fig. 4(b), the core can be approximated simply by a straight line connecting the vortex centers on the top and bottom surfaces. In this model, the fundamental gyrotropic mode $V_{0}$ is a simple circulation of this line around the central position, as sketched in Fig. 5(a). Higher-order modes of such a circulating mode are sketched in the other panels (c), (d). In these modes, the vortex core also oscillates on a circular orbit on the surfaces, but the amplitude of this oscillation varies along the thickness. The first order mode $V_{1}$ contains one node of this oscillation in the center, while the mode $V_{2}$ is characterized by two such nodes. Obviously, these higher-order modes can only occur in 3D samples of sufficiently large thickness to allow for variations of the magnetization dynamics along the vortex core. This is consistent with the findings of Boust et al. ${ }^{26}$ who found that a higher-order vortex core mode occurs in sufficiently thick cylindrical permalloy nanodots. Even though in practice the modes identified as $V_{1}$ and $V_{2}$ have a much more complicated spatial distribution compared with the simple sketch in Fig. 5, this simple model captures most of the basic features of the 3D vortex modes which will be described in detail in the rest of this section.

Figure 6(a) shows a snapshot of both the top and bottom surface for the $V_{0}$ mode resolved for the 60-nm-thick sample. The frequency of this mode is $1.1 \mathrm{GHz}$. Note that what is shown here is only the dynamic magnetization, i.e., the change of magnetization from equilibrium. The equilibrium position of the vortex core is located between the yellow (bright) and blue (dark) dots shown in Fig. 6(a). The core is displaced towards the yellow (bright) dot, in the direction along the line connecting the yellow (bright) and blue (dark) dots. In the direction opposite to the one the core is displaced to, there is a region where the $z$ component of the magnetization decreases correspondingly, which appears as a blue

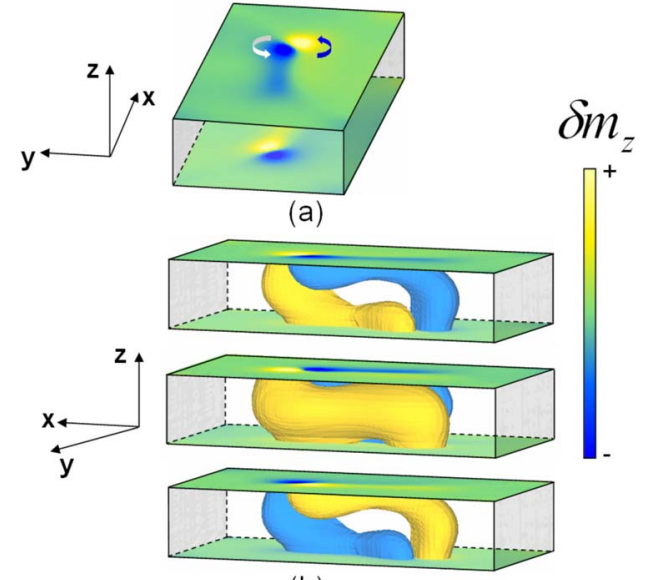

(b)

FIG. 6. (Color online) Illustration of the $V_{0}$ mode resolved for the 60-nm-thick sample. Only the variations of the $z$ component are shown. (a) Snapshot of the dynamical magnetization on the top and bottom surface. The blue (dark) and white arrow indicate the rotational direction of the blue (dark) and yellow (bright) spot on the top surface. On the bottom surface, the rotational direction is the same but with a different phase. (b) Three snapshots of the dynamical magnetization inside the sample. The first one is taken at the same moment as (a). Two isosurfaces with constant absolute value of $\delta m_{z}$, positive (yellow/bright) and negative (blue/dark), are shown in each snapshot.

(dark) dot in the figure. The arrows indicate the rotational direction of the dots, which is counterclockwise in this case. This is consistent with the prediction of Eq. (4), since here the core is polarized in positive $z$ direction, as shown in Fig. 4. At the surfaces, this mode is recognized to be the gyrotropic mode of the vortex. A significant difference with respect to the 2D case can be seen in Fig. 6(a): The vortex core gyration occurs at different phases on the top and bottom surface, with the top surface in this case lagging behind by about $90^{\circ}$ with respect to the bottom surface.

The interior profile of this mode is shown in three snapshots in Fig. 6(b). Two isosurfaces with positive and negative constant value of the $z$ component of the dynamic part of the magnetization $\delta m$ are displayed. Hence, the isosurfaces represent areas of constant oscillation magnitude. In each of these snapshots, the yellow (bright) surface describes the regions in which the $z$ component of the magnetization increases by a specific amount, while the blue (dark) surface shows the regions where it decreases by the same amount. In the central part of the sample, the $V_{0}$ mode is located near the Bloch part of the asymmetric domain wall. In this sense, it can be considered as a domain-wall oscillation mode. Since this oscillation is also coupled to the gyrotropic motion of the vortices on the surfaces, it is more convenient to interpret this as a coherent gyration of the stretched vortex core shown in Fig. 4(b). Analogous to the previously described effects on the surface, where the rotation of the vortex core results in one spot where $m_{z}$ increases and a second one where it decreases, a displacement of the stretched vortex core leads to two "tubes" in this isosurface representation, one of which denotes the increase and the other the decrease of $m_{z}$. Thus, 


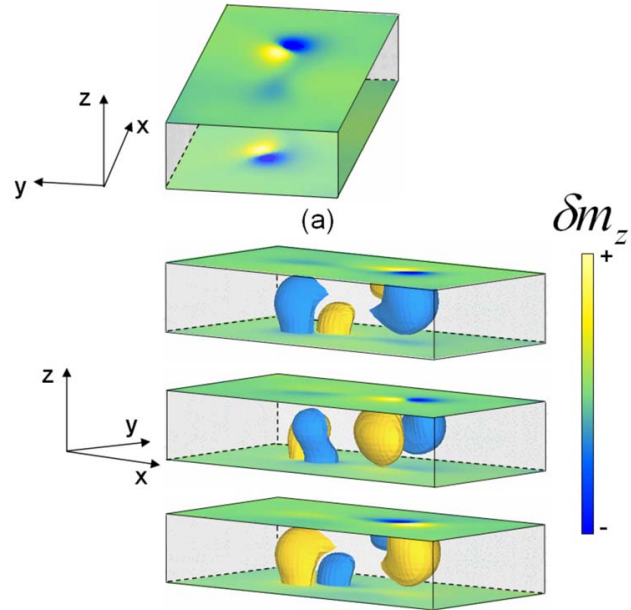

(b)

FIG. 7. (Color online) Illustration of the $V_{1}$ mode resolved for the 60-nm-thick sample. (a) Snapshot of the dynamic magnetization on top and bottom surfaces. (b) Three snapshots of the dynamic magnetization inside the sample with the first one at the same moment as (a). Two isosurfaces with constant absolute value of $\delta m_{z}$, positive (yellow/bright) and negative (blue/dark), are shown in each snapshot.

the two blue (dark) spots and the two yellow (bright) spots on the top and bottom surfaces are connected naturally by the yellow (bright) and the blue (dark) pipelike regions. The third snapshot is taken half a period later, when the oscillation has the opposite phase with respect to the first snapshot. The time difference between the first and the third snapshots is half the period of oscillation, while the second snapshot is taken at a moment in between. In this representation of the $V_{0}$ mode, the yellow (bright) and the blue (dark) tubes are constantly revolving around each other, with the sense of rotation determined by the core magnetization direction.

For the 60-nm-thick sample, the simulations yield another mode with a slightly larger frequency of $1.3 \mathrm{GHz}$. This mode is labeled $V_{1}$, and it can be regarded as a variant of the fundamental gyrotropic mode $V_{0}$. At the surfaces, this mode also appears as a gyrotropic mode, albeit with a phase difference on the top and the bottom surface. Three snapshots inside the sample are shown in Fig. 7(b) using an isosurface representation as was done previously in the description of the $V_{0}$ mode. Again, the difference in time between the first and the third snapshot of Fig. 7(b) is half a period of the oscillation and the second frame shows the situation at an intermediate time. It can be seen that the variations of the surface magnetization [represented by the yellow (bright) and the blue (dark) spots] extend deep into the sample, similar to the case of the $V_{0}$ mode. The difference here is that these oscillating areas are not connecting the opposite surfaces, as it was previously the case with the "tubes." The oscillating regions are mostly localized around the surface, and are gyrating about the equilibrium position of the vortex at the surface. In this mode, the oscillations are most pronounced near the surface, while in the center of the sample there is a region of small amplitude. These features are consistent with the schematic representation of the mode $V_{1}$ as

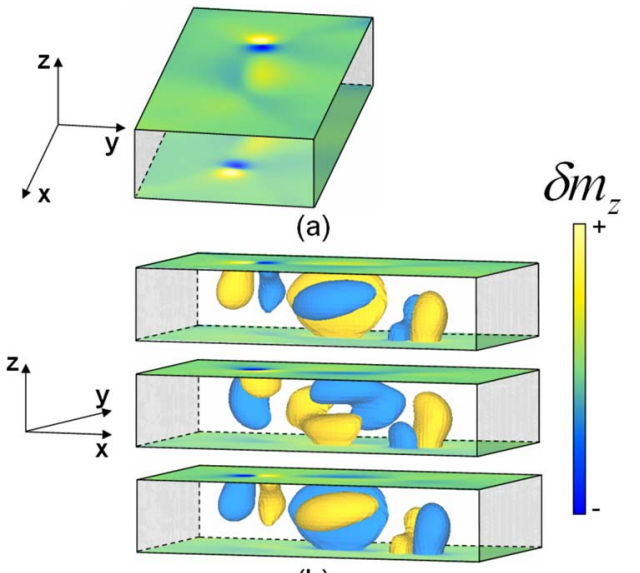

(b)

FIG. 8. (Color online) Illustration of the $V_{2}$ mode resolved for the 80-nm-thick sample. (a) Snapshot of the dynamic magnetization on top and bottom surface. (b) Three snapshots of the dynamic magnetization inside the sample with the first one at the same moment as (a). Two isosurfaces with constant absolute value of $\delta m_{z}$, positive (yellow/bright) and negative (blue/dark), are shown in each snapshot.

shown in Fig. 5(b). The oscillation amplitude of the $V_{1}$ mode is much smaller than it is in the $V_{0}$ mode. In the 60-nm-thick sample, it is about half as large.

A third mode of the vortex core oscillation is displayed in Fig. 8. In this case, the $80-\mathrm{nm}$ sample has been chosen to illustrate the profile of this mode, because it is better resolved in particles of larger thickness. This is the secondorder vortex mode $V_{2}$, and it has a frequency of $1.9 \mathrm{GHz}$. Similar to the two previous modes, it appears as the rotation of the vortex core at the surfaces [see Fig. 8(a)]. In Fig. 8(b), the same representation with isosurfaces is used to display the evolution of the mode profile over half an oscillation period. The mode has a complicated internal structure, which consists of localized regions with pronounced oscillations near the surface vortices (as was the case in the mode $V_{1}$ ) and a pronounced oscillation of the central region. These three different regions are separated by areas with oscillations with low-amplitude and they are gyrating around the equilibrium position of the stretched vortex core. The central region oscillates almost with opposite phase with respect to the surface oscillations. This corresponds to the schematic representation of this mode as shown in Fig. 5(c). Although the details of this mode are very complicated, the basic features of three strongly oscillating regions separated by two low-amplitude regions (nodes) are well reproduced by this simple model.

\section{A. Comparison of the vortex modes}

All three modes discussed above are related to the rotation of the vortex core, at least from a view on only the top and bottom surfaces. The $V_{0}$ mode has the lowest frequency and the simplest structure inside the sample. This mode is analogous to the gyrotropic mode occurring in 2D systems and can be considered as the rotational mode of the stretched 
(a)

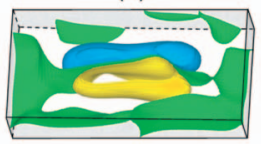

$\delta m_{z}$ (b)

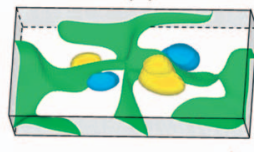

$z^{*}$

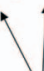

\section{$\varphi^{y}$}

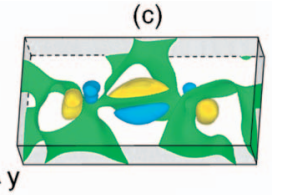

FIG. 9. (Color) Perspective view on the internal oscillations of (a) the $V_{0}$ mode, (b) the $V_{1}$ mode, and (c) the $V_{2}$ mode with isosurface representation. In addition to the regions of strong variations in $m_{z}$ shown as previously with yellow and blue surfaces, the isosurfaces $\delta m_{z}=0$ are displayed in green.

vortex core in 3D prisms. The two other modes $V_{1}$ and $V_{2}$, have higher frequencies and acquire more complex structures inside the sample. These modes have been interpreted as higher-order modes according to the sketch in Fig. 5.

In one-dimensional (1D) and 2D systems, higher-order modes are often characterized by the existence of nodes or nodal lines. A straightforward extension of this concept to the $3 \mathrm{D}$ case is the introduction of nodal planes, i.e., the identification of surfaces inside the volume, which are located between the oscillating regions, and which describe areas, where the magnetization remains constant in time and space for a specific frequency. However, we found that such nodal surfaces could not be identified in our simulations, at least not in the strict sense as it is applicable in simpler systems. Although surfaces with zero fluctuation $\delta m=0$ can be easiliy determined at any point in time for each mode, it is found that these curved surfaces constantly change their position and shape. Therefore, no region can be found, where the magnetization remains exactly constant over the entire oscillation period. As a result, the isosurfaces $\delta m=0$ obtained in each simulated frame cannot be defined-strictly speaking - as nodal surfaces of the mode. It is nevertheless helpful to display these isosurfaces with $\delta m=0$, since they represent at least a good approximation to nodal surfaces. This is shown in Fig. 9, where snapshots of the $\delta m_{z}=0$ isosurfaces are displayed for the three vortex modes.

The "nodal" surfaces $\delta m_{z}=0$ separate the oscillating regions with positive variations from those with negative variations. It is evident that with increasing order (i.e. from the mode $V_{0}$ to the mode $V_{2}$ ) the number of $\delta m_{z}=0$ isosurfaces separating such regions increases. In this sense, the situation is analogous to the well-known $2 \mathrm{D}$ case. The increase in the number of nodal surfaces is consistent with the observed increase of the mode frequency. This effect has also been observed in studies of higher-order excitation modes in 2D disks. ${ }^{2,16}$ The isosurface representation of Fig. 9 helps localizing the approximate position of the nodes and thereby corroborates the applicability of the simple "string" model of Fig. 5.

\section{B. Thickness dependence}

Finite-size effects are decisive for the formation of the magnetic flux-closure patterns. We can thus expect that the particle size and shape also influence the dynamic behavior

of the magnetization. In particular, the thickness dependence of the observed modes and their frequencies is important when 3D effects of the magnetization dynamics are to be studied. For this purpose the various modes have been analyzed in three different samples of the same material and aspect ratio, but different size. The size dependence is in this case effectively a dependence on the thickness, since the in-plane part of the magnetic flux closure pattern is largely invariant in the three samples. On the other hand, variations of only the thickness while keeping the in-plane sample size constant would correspond to a change of aspect ratio. This would lead to differences between the ground-state configurations of the different samples, thus making it much more difficult to directly compare the respective modes in the different samples. We hence kept the aspect ratio constant and varied only the size. The thickness of the three samples is of 60,70 , and $80 \mathrm{~nm}$.

The $V_{0}$ mode is observed in all three samples. In the previous section, the $V_{0}$ mode has been identified as the $3 \mathrm{D}$ counterpart of the gyrotropic vortex mode known from 2D thin-film elements. Remarkably, the frequency of this mode is the same for the three samples $(1.1 \mathrm{GHz})$. This is consistent with previous studies ${ }^{17,18,41,42}$ of this mode in the $2 \mathrm{D}$ case, where it has been shown that the gyrotropic motion depends on the aspect ratio of the elements.

The $V_{2}$ mode is also observed in all three samples, but with varying frequency. For the 80-, 70-, and 60-nm-thick samples, the frequencies are $1.9,2.2$, and $2.9 \mathrm{GHz}$, respectively. This size dependence of the frequency can be attributed to the different length of the antinodal segments along the vortex core. The length of the domain wall [or the length of the tube shown in Fig. 4(b)] should be approximately proportional to the length of the sample. A shorter wall length in a smaller sample will cause larger exchange energy, thus higher frequency, with the same number of nodes. This is in agreement with the simple string model of Fig. 5, where a reduction of the layer thickness is intuitively expected to increase the frequency. The decrease of frequency of a higher-order vortex core mode with increasing thickness has also been reported by Boust et al. ${ }^{26}$ who performed similar 3D micromagnetic simulations on a somewhat simpler magnetic object with vortex configuration.

The $V_{1}$ mode is observed in the $60-\mathrm{nm}$ - and in the 70-nm-thick sample, but not in the 80-nm-thick one. The frequency decreases from $1.3 \mathrm{GHz}$ in the $60-\mathrm{nm}$ sample to $1.2 \mathrm{GHz}$ in the $70-\mathrm{nm}$ sample. It can be assumed that the mode also occurs in the $80-\mathrm{nm}$ sample, but its frequency is probably too close to the $V_{0}$ mode to be resolved properly in the simulations. Similar difficulties in separating almost degenerate modes have been reported also in related experimental studies on 2D systems. ${ }^{2,16}$ Note that the amplitude of the oscillations found in the $V_{0}$ mode is always significantly larger than the amplitude of the oscillations found in the $V_{1}$ mode. Therefore, a superposition of these two modes in the case of almost identical frequency might not be detectable. A systematic study on the size dependence would obviously require the analysis of a larger number of samples. However, such a systematic analysis is difficult to obtain numerically. Since a high spatial resolution (discretization density) is necessary throughout the sample to obtain accurate and well- 
resolved results, the number of required discretization points increases approximately with the third power of the size (edge length). Thus, the size of the three-dimensional objects which can be calculated is limited by the drastically increasing requirements of computer resources, and we currently cannot go significantly beyond size of the $400 \times 200$ $\times 80 \mathrm{~nm}^{3}$ sample for high-resolution simulations as they are required in this study.

It is also worthwhile to point out that in our calculations, all three gyrotropic modes can be excited by both in-plane and out-of-plane pulse. This differs from the situation in 2D symmetric vortex structures, in which the gyrotropic mode can only be excited by an in-plane pulse, as an out-of-plane pulse cannot displace the vortex core. ${ }^{19}$ In our samples, however, due to the intrinsic asymmetry of the ground state, even a homogeneous out-of-plane pulse can exert a torque to the vortex core.

\section{Relative phase of the vortex gyration at the surfaces}

An interesting feature of the $3 \mathrm{D}$ vortex modes is that the vortices generally rotate with a different phase on the top and bottom surfaces. In the case of the $V_{0}$ mode in the $60-\mathrm{nm}$ sample, the rotation of the core on the top surface is approximately $90^{\circ}$ behind that of the bottom one (defined as a phase difference of $+90^{\circ}$ ). Such a phase difference is also observed in the same sample for the $V_{1}$ mode, but the relative phase of the vortex rotation on the top and bottom surface is different. As shown in Fig. 7(a), the top surface is now approximately $90^{\circ}$ ahead of the bottom surface in this mode. Hence, the relative phase of the oscillations in the $V_{0}$ and in the $V_{1}$ mode changes by about $180^{\circ}$. This is in agreement with the interpretation according to the simple model shown in Fig. 5, where the phase difference of the oscillations at the surfaces increases by $180^{\circ}$ in the $V_{1}$ mode with respect to the $V_{0}$ mode. In this case, the variations of the phase shift with increasing order are rather well reproduced by this simple model. In spite of this qualitative agreement, it appears that the phase relation between the vortex rotation on the top and bottom surface is in fact very complicated. For example, we have found different relative phases for different variants of the $V_{0}$ mode. This mode is resolved in six different cases (two for each sample: one from the in-plane pulse and another from the out-of-plane pulse), each of which displays different phase relations even though the mode profile inside the wall is similar for all those modes. Three of them have a phase difference close to $90^{\circ}$, one a difference of about $45^{\circ}$, another one a difference of about $30^{\circ}$ and yet another one a difference close to $130^{\circ}$. In principle, the frequency of those modes should vary with the relative phase change, but the small frequency difference might be below our numerical resolution $(0.1 \mathrm{GHz})$. In the case of the $V_{1}$ mode, the relative phase difference of the core rotation is about $-60^{\circ}$ in the $70 \mathrm{~nm}$ sample, while a phase difference of about $130^{\circ}$ is found in the case of the $V_{2}$ mode in the $80 \mathrm{~nm}$ sample. The relative phase difference of the core rotation on the top and bottom surfaces certainly plays an important role for adapting the two surfaces to the inner structure of the modes. Unfortunately, not enough data are collected for a quantitative analysis.
The phase difference of the vortex gyration at the surface observed in the simulations of the individual 3D vortex modes can obviously not be fully explained by the simple model of Fig. 5. Even though this model can greatly help in understanding some basic features of these new 3D gyrating vortex modes, it is important to keep its limitations in mind. The model can be justified by the fact that-in the static structure-the vortex cores directly connect to the Bloch part of the asymmetric wall. Hence, the vortex motions on the two surfaces are not independent. However, the magnetic structure in the center of the sample has features, which differ quite significantly from those of an ordinary vortex core. Thus, the interpretation of the tubelike region as a single vortex core can be regarded as a drastic simplification of the actual magnetic structure. The model completely neglects the asymmetry of the domain wall in the central part of the sample (as shown in Fig. 3). In fact, the amplitude of the oscillation on opposite sides of the domain wall is different for all the vortex modes, as can be seen by the different shape of two isosurfaces with the same absolute value and by the curved nodal planes shown in Fig. 9. This is not surprising, because the magnetization distribution of the wall in equilibrium is asymmetric. The vortex modes can alternatively be considered as oscillation modes of the asymmetric Bloch wall, the frequency of which is determined by the internal structure. In this picture, the vortex oscillations on the surfaces merely occur as a result of the domain wall oscillation.

\section{HIGHER-FREQUENCY MODES: EDGES, CORNERS AND CLOSURE DOMAIN WALLS}

In addition to the vortices and the asymmetric Bloch wall, other regions of inhomogeneous magnetization such as the corners and the $90^{\circ}$ domain walls display distinct dynamic properties. This is also known from previous studies on 2D systems, for example, in vortex structures found in thin square elements. ${ }^{24}$ Similar to the simulation results reported for square thin elements with vortex configuration, ${ }^{24}$ some characteristic modes mainly localized at corners and $90^{\circ}$ domain walls are observed in our 3D samples as well. These three-dimensional modes, however, have features differing from the $2 \mathrm{D}$ case regarding both the amplitude and phase.

Figure 10 shows the profile of the mode marked $E_{1}$, which has a frequency of $3.6 \mathrm{GHz}$ for the 60 -nm-thick sample. In Fig. 10(a), an isosurface representation of the amplitude of the variations of the $z$ component is displayed, regardless of the phase. This mode is mainly localized along the four short edges of the prism. The oscillations at these edges have different amplitude, although the tipping pulse used to excite this mode is applied homogeneously in space (along the $z$ direction in this case). Such differences in amplitude in the four corners are not surprising, given the overall asymmetry of the ground state magnetization pattern. Moreover, already the static structure removes the degeneracy of the corners. As the magnetization in soft-magnetic materials tends to align parallel to surfaces and edges in order to minimize surface charges, the magnetization partially also aligns with the short edges along the $z$ axis. This align- 


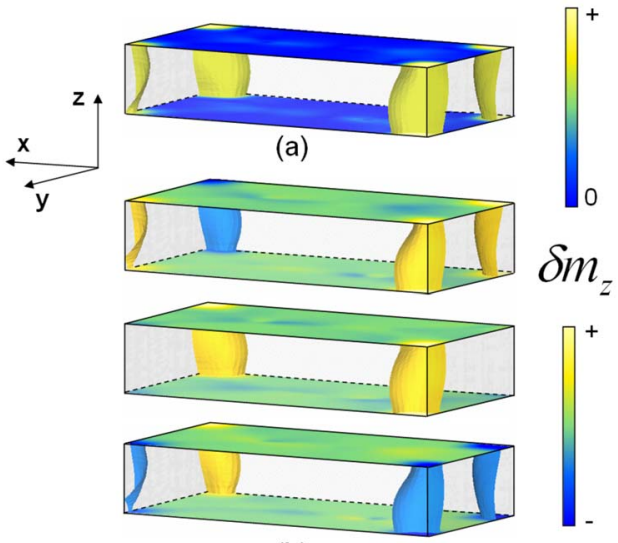

(b)

FIG. 10. (Color online) Illustration of the $E_{1}$ mode resolved for the 60-nm-thick sample. (a) Power distribution of the mode regardless of the phase. (b) Three snapshots of the dynamical magnetization inside the sample. Two isosurfaces with constant absolute value of $\delta m_{z}$, positive (yellow/bright) and negative (blue/dark), are drawn in each snapshot. Note that (a) and (b) use a different color bar (gray scale). The coordinate system applies to both (a) and (b).

ment can be either in the positive or in the negative $z$ direction, giving rise to a large number of almost degenerate ground-state configurations, unlike in the $2 \mathrm{D}$ case where the magnetization is always aligned in the $x y$ plane even at the corners. Different orientations of the $z$ component at the edges are expected to affect the relative phase of their oscillation modes. A detailed analysis of these effects, however, is going beyond the scope of this study.

The $E_{1}$ mode not only shows a variation of amplitude, but also a phase decoherence of the four edges, as can be seen from the three snapshots of this mode shown in Fig. 10(b). The four corners exhibit a complex phase relation among them, instead of being all in phase as would be the case in square thin film element with a symmetric vortex structure excited by a short, homogeneous field pulse. ${ }^{24}$

For the same sample, we also found at least one further mode of the same type as the $E_{1}$ mode, with a slightly different frequency $(3.7 \mathrm{GHz}$ for the 60-nm sample) and with different relative phases of the four edges. This phase dependence of the frequency can be attributed to dipolar interactions between the edges.

Figure 11 shows a different edge mode $\left(E_{2}\right)$ with a considerably higher frequency $(5.7 \mathrm{GHz}$ for the 60 -nm-thick sample). Although this mode displays oscillations at the edges, similar to the $E_{1}$ mode, the power of this mode also extends into the four $90^{\circ}$ short domain walls of the closure domains, as shown in Figs. 11(a) and 11(c). Again, an asymmetry among the four corners is observed. Three snapshots of this mode are shown in Fig. 11(b). To describe this mode, it is sufficient to focus on the short perpendicular edge on the left-front part of the sample. In the first snapshot, the negative region (blue/dark) is mainly located at the lower corner and the positive region (yellow/bright) is mainly at the upper part of the $90^{\circ}$ domain wall. In the second snapshot, the negative region has shifted towards the upper corner of the edge, and the positive region to the lower part of the wall.

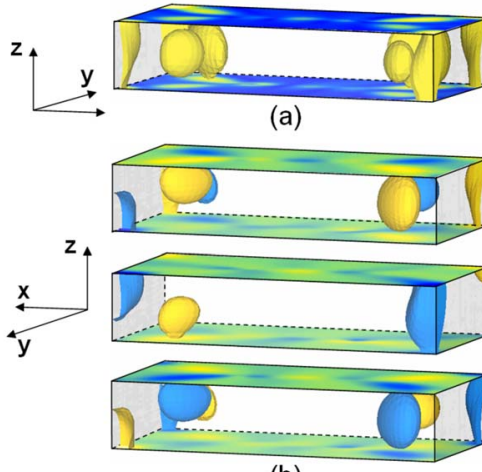

(b)

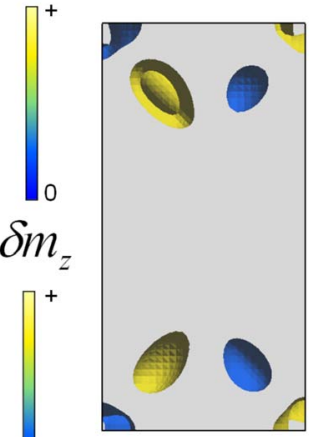

(c)
FIG. 11. (Color online) Illustration of the $E_{2}$ mode resolved for the 60-nm-thick sample. (a) Power distribution of the mode regardless of the phase. (b) Three snapshots of the dynamic magnetization inside the sample. Isosurfaces with constant absolute value of $\delta m_{z}$, positive (yellow/bright) and negative (blue/dark) are drawn in each snapshot. Note that (a) and (b) use a different color bar (gray scale). The in-plane distribution of the oscillating regions is shown with a top view in frame (c).

The third snapshot is a half period after the first one, and it therefore displays the situation at opposite phase. In this mode, the corner and its neighboring wall seem to form a circuit inside the sample along which they exchange their phase. This circuit connects the lower and the upper corner of the edge with the upper and the lower part of the adjacent $90^{\circ}$ domain wall. The other perpendicular edges show similar behavior. This way of connecting pulsating regions is made possible by the increased number of degrees of freedom resulting from the non-negligible thickness of the sample. Indeed, for a similar mode observed in a 2D square vortex, each of the four corners and its neighboring wall have been found to oscillate with exactly opposite phase, forming a well-defined nodal line in-between. ${ }^{24}$ In our 3D case, however, the corners and the walls are oscillating with a more complicated phase relation. It appears that in the 3D case, the "circulating" phase has lower exchange energy as compared to the situation where the corners and its neighboring $90^{\circ}$ domain wall are oscillating with exactly opposite phase.

For the same sample a further, similar mode with even higher frequency $(6.7 \mathrm{GHz})$ is resolved and shown in Fig. 12. By comparing the power distribution on the surface [Fig. 12(a)] to Fig. 11(a), this mode can be categorized as a corner mode $\left(C_{1}\right.$, not shown in the spectrum of Fig. 1$)$ rather than an oscillation of the edges and domain walls. Especially by looking at the two front edges, a nodal area seems to exist in the middle between the upper and the lower part of the edges. The mode also extends into the region of the $90^{\circ}$ wall, similar to the $E_{2}$ mode, but with a smaller relative amplitude. The oscillations of the $90^{\circ}$ domain wall are localized in the interior part of the sample, instead of the surface regions of the $90^{\circ}$ walls as in the case in the $E_{2}$ mode. Figure $12(\mathrm{~b})$ shows three snapshots of this mode. In the first snapshotagain only focusing on the left-front edge-the upper and lower parts are oscillating with different phase, but not completely opposite. The upper part has stronger amplitude than 


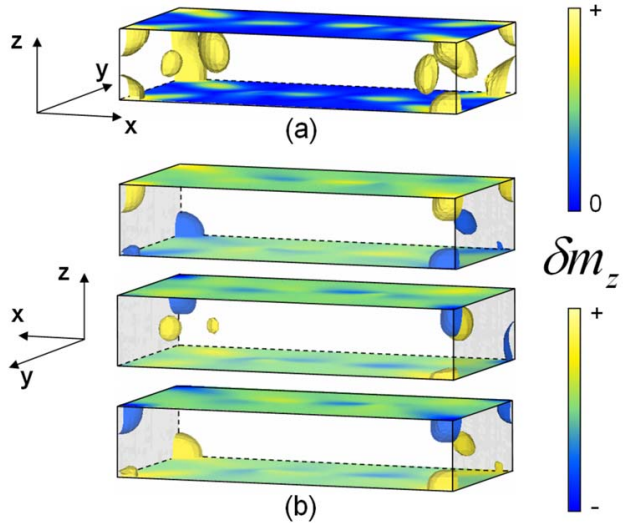

FIG. 12. (Color online) Illustration of the $C_{1}$ mode resolved for the 60-nm-thick sample. (a) Power distribution of the mode regardless of the phase. (b) Three snapshots of the dynamical magnetization inside the sample with emphasis only on the left-front corner. Two isosurfaces with constant absolute value of $\delta m_{z}$, positive (yellow/bright) and negative (blue/dark), are drawn in each snapshot. Note that (a) and (b) use a different color bar (grayscale).

the lower part. In the second snapshot, the oscillation is mainly located in the inner part of the $90^{\circ}$ wall. It should be noted that compared with the previous modes, the $C_{1}$ mode does not appear to be perfectly resolved in the simulations, which is probably due to a superposition of modes which are almost degenerate. In a first approximation, this mode can be regarded as a corner mode in which a standing wave is formed along the thickness direction. However, it displays more complicated features. Similar to the $E_{2}$ mode shown in Fig. 11, this mode also has a circulating phase, which changes along a path between the corners and the adjacent $90^{\circ}$ domain wall. The difference here is that the path is shorter, connecting the corners directly to the central part of the domain wall region without passing through the sample surfaces. The upper and lower part of the corner exchange their phase in a circular way via the central part of the $90^{\circ}$ domain wall instead of oscillating with opposite phase, which again lowers the exchange energy. The special phase relation observed in the modes $E_{2}$ and $C_{1}$ is yet another direct consequence of the $3 \mathrm{D}$ character of the system studied here.

The list of 3D modes reported in this work is certainly not complete. As can be seen in Fig. 1, a number of further peaks can be detected in the power spectrum, e.g., in the frequency range between the $E_{1}$ and the $E_{2}$ mode. The oscillations at these frequencies are much more complicated than the modes described previously. They appear to be complex, coupled oscillations involving the edges, the central domain wall, and the domain walls of the closure domains, which oscillate with nontrivial phase relations. Many of these additional oscillations can thus be regarded as hybrid modes consisting of combinations of the abovementioned modes.

\section{CONCLUSIONS}

In conclusion, we presented micromagnetic simulations of magnetic normal modes in 3D Permalloy prisms with asymmetric vortex-state configurations. Spatially resolved Fourier analysis of the complicated dynamics has enabled us to identify various characteristic modes in the 3D magnetic structures. The particular role played by the three-dimensionally distorted vortex core (or, equally, by the asymmetric Bloch wall) has been studied in detail. Our simulations anticipate the existence of previously unreported higher-order modes of the gyrotropic vortex excitation. Specific predictions are made concerning the variations in frequency of these modes with changing particle size. While the frequency of the fundamental gyrotropic mode $V_{0}$ does not depend on the sample size, the higher-order modes $V_{1}$ and $V_{2}$ show a significant increase in frequency in smaller samples. This behavior of the peaks in the power spectrum should be rather easily observable in experiments, e.g., by FMR measurements, while an experimental verification of the calculated complex internal oscillation patterns might be much more challenging. In addition to the vortex modes, we found higher-frequency oscillations of the sample edges and corners and of the $90^{\circ}$ closure domain walls. Although these modes share some basic characteristics with the previously reported corresponding $2 \mathrm{D}$ modes, they have several new features and differences which arise from the increased degrees of freedom along the sample thickness. Our studies on rectangular bars clearly show that the transition from $2 \mathrm{D}$ to $3 \mathrm{D}$ micromagnetic configurations is not straightforward and has a strong influence on the dynamic behavior. The asymmetric micromagnetic ground state in the 3D system introduces particular complications, which will require further in-depth analyses.

\section{ACKNOWLEDGMENTS}

We would like to thank S. Gliga for the careful reading of the manuscript and for several valuable suggestions and Y. Liu and S. Gliga for their very helpful hints and support concerning the graphical visualization.
${ }^{1}$ B. McMichael and M. D. Stiles, J. Appl. Phys. 97, 10J901 (2005).

${ }^{2}$ M. Buess, R. Höllinger, T. Haug, K. Perzlmaier, U. Krey, D. Pescia, M. R. Scheinfein, D. Weiss, and C. H. Back, Phys. Rev. Lett. 93, 077207 (2004).

${ }^{3}$ C. Mathieu, J. Jorzick, A. Frank, S. O. Demokritov, A. N. Slavin, B. Hillebrands, B. Bartenlian, C. Chappert, D. Decanini, F.
Rousseaux, and E. Cambril, Phys. Rev. Lett. 81, 3968 (1998).

${ }^{4}$ J. Jorzick, S. O. Demokritov, B. Hillebrands, M. Bailleul, C. Fermon, K. Y. Guslienko, A. N. Slavin, D. V. Berkov, and N. L. Gorn, Phys. Rev. Lett. 88, 047204 (2002).

${ }^{5}$ J. P. Park, P. Eames, D. M. Engebretson, J. Berezovsky, and P. A. Crowell, Phys. Rev. Lett. 89, 277201 (2002).

${ }^{6}$ C. Bayer, J. P. Park, H. Wang, M. Yan, C. E. Campbell, and P. A. 
Crowell, Phys. Rev. B 69, 134401 (2004).

${ }^{7}$ M. Yan, H. Wang, P. A. Crowell, and C. E. Campbell, Condens. Matter Theor. 20, 251 (2005).

${ }^{8}$ K. Y. Guslienko, R. W. Chantrell, and A. N. Slavin, Phys. Rev. B 68, 024422 (2003).

${ }^{9}$ M. Grimsditch, G. K. Leaf, H. G. Kaper, D. A. Karpeev, and R. E. Camley, Phys. Rev. B 69, 174428 (2004).

${ }^{10}$ M. Grimsditch, L. Giovannini, F. Montoncello, F. Nizzoli, G. K. Leaf, and H. G. Kaper, Phys. Rev. B 70, 054409 (2004).

${ }^{11}$ Y. Acremann, C. H. Back, M. Buess, O. Portmann, A. Vaterlaus, D. Pescia, and H. Melchior, Science 290, 492 (2002).

${ }^{12}$ V. Novosad, M. Grimsditch, K. Y. Guslienko, P. Vavassori, Y. Otani, and S. D. Bader, Phys. Rev. B 66, 052407 (2002).

${ }^{13}$ B. A. Ivanov and C. E. Zaspel, Appl. Phys. Lett. 81, 1261 (2002).

${ }^{14}$ L. Giovannini, F. Montoncello, F. Nizzoli, G. Gubbiotti, G. Carlotti, T. Okuno, T. Shinjo, and M. Grimsditch, Phys. Rev. B 70, 172404 (2004).

${ }^{15}$ B. A. Ivanov and C. E. Zaspel, Phys. Rev. Lett. 94, 027205 (2005).

${ }^{16}$ M. Buess, T. Haug, M. R. Scheinfein, and C. H. Back, Phys. Rev. Lett. 94, 127205 (2005).

${ }^{17}$ K. Y. Guslienko, X. F. Han, D. J. Keavney, R. Divan, and S. D. Bader, Phys. Rev. Lett. 96, 067205 (2006).

${ }^{18}$ V. Novosad, F. Y. Fradin, P. E. Roy, K. S. Buchanan, K. Y. Guslienko, and S. D. Bader, Phys. Rev. B 72, 024455 (2005).

${ }^{19}$ X. Zhu, Z. Liu, V. Metlushko, P. Grütter, and M. R. Freeman, Phys. Rev. B 71, 180408(R) (2005).

${ }^{20}$ J. P. Park, P. Eames, D. M. Engebretson, J. Berezovsky, and P. A. Crowell, Phys. Rev. B 67, 020403(R) (2003).

${ }^{21}$ S.-B. Choe, Y. Acremann, A. Scholl, A. Bauer, A. Doran, J. Stöhr, and H. A. Padmore, Science 304, 420 (2004).

${ }^{22}$ K. Perzlmaier, M. Buess, C. H. Back, V. E. Demidov, B. Hillebrands, and S. O. Demokritov, Phys. Rev. Lett. 94, 057202 (2005).

${ }^{23}$ J. Raabe, C. Quitmann, C. H. Back, F. Nolting, S. Johnson, and C.
Buehler, Phys. Rev. Lett. 94, 217204 (2005).

${ }^{24}$ M. Yan, G. Leaf, H. Kaper, R. Camley, and M. Grimsditch, Phys. Rev. B 73, 014425 (2006).

${ }^{25}$ J. Podbielski, F. Giesen, and D. Grundler, Phys. Rev. Lett. 96, 167207 (2006).

${ }^{26}$ F. Boust and N. Vukadinovic, Phys. Rev. B 70, 172408 (2004).

${ }^{27}$ N. Vukadinovic and F. Boust, Phys. Rev. B 75, 014420 (2007).

${ }^{28}$ V. S. Pribiag, I. N. Krivorotov, G. D. Fuchs, P. M. Braganca, O. Ozatay, J. C. Sankey, D. C. Ralph, and R. A. Buhrman, Nat. Phys. 3, 498 (2007).

${ }^{29}$ R. Hertel, O. Fruchart, S. Cherifi, P.-O. Jubert, S. Heun, A. Locatelli, and J. Kirschner, Phys. Rev. B 72, 214409 (2005).

${ }^{30}$ R. Hertel and H. Kronmüller, Phys. Rev. B 60, 7366 (1999).

${ }^{31}$ R. Hertel, J. Appl. Phys. 90, 5752 (2001).

${ }^{32}$ R. Hertel and C. M. Schneider, Phys. Rev. Lett. 97, 177202 (2006).

${ }^{33}$ D. R. Fredkin and T. R. Koehler, IEEE Trans. Magn. 26, 415 (1990).

${ }^{34}$ A. C. Hindmarsh, in Scientific Computing (North-Holland, Amsterdam, 1983), pp. 55-64.

${ }^{35}$ See EPAPS Document No. E-PRBMDO-76-035733 for various movies in avi-format showing the modes discussed in this article. For more information on EPAPS, see http://www.aip.org/ pubservs/epaps.html

${ }^{36}$ A. E. LaBonte, J. Appl. Phys. 40, 2450 (1969).

${ }^{37}$ A. Hubert, Phys. Status Solidi 32, 519 (1969).

${ }^{38}$ A. S. Arrott and T. L. Templeton, Physica B 233, 259 (1997).

${ }^{39}$ The visualization of the magnetic domains and the oscillation modes is done with GMV, http://www-xdiv.lanl.gov/XCM/gmv/ GMVHome.html

${ }^{40}$ A. A. Thiele, Phys. Rev. Lett. 30, 230 (1973).

${ }^{41}$ K. Y. Guslienko, B. A. Ivanov, V. Novosad, Y. Otani, H. Shima, and K. Fukachimi, J. Appl. Phys. 91, 8037 (2002).

${ }^{42}$ C. E. Zaspel, B. A. Ivanov, J. P. Park, and P. A. Crowell, Phys. Rev. B 72, 024427 (2005) 